This is a pre-copy-editing, author-produced PDF of an article accepted for publication in "The Computer Journal" following peer review.

The definitive publisher-authenticated version:

\title{
Measuring and Evaluating the Visual Complexity of Chinese Ink Paintings
}

Fan, Zhen-Bao; LI, Yina; Zhang, Kang; Yu, Jinhui; Huang, Mao Lin

, March 2021, Pages 1-13

The article is available online at:

https://academic.oup.com/comjnl/issue 


\section{The Computer Journal}

\section{Measuring and Evaluating the Visual Complexity of Chinese Ink Paintings}

\begin{tabular}{|c|c|}
\hline Journal: & The Computer Journal \\
\hline Manuscript ID & COMPJ-2020-04-0344.R2 \\
\hline Manuscript Type: & Original Article \\
\hline $\begin{array}{r}\text { Date Submitted by the } \\
\text { Author: }\end{array}$ & 02-Mar-2021 \\
\hline Complete List of Authors: & $\begin{array}{l}\text { Fan, Zhenbao; The University of Texas at Dallas, Department of } \\
\text { Computer Science } \\
\text { Li, Yina; University of Technology Sydney, School of Computer Science } \\
\text { Zhang, Kang; The University of Texas at Dallas, Department of } \\
\text { Computer Science } \\
\text { Yu, Jinhui; Zhejiang University, College of Computer Science and } \\
\text { Technology } \\
\text { Huang, Mao Lin; University of Technology Sydney, School of Computer } \\
\text { Science }\end{array}$ \\
\hline Key Words: & $\begin{array}{l}\text { Paintings, Complexity, Regression Model, Visual Rhetoric, Visual- } \\
\text { Information Processing }\end{array}$ \\
\hline
\end{tabular}

\section{SCHOLARONE" \\ Manuscripts}




\title{
Measuring and Evaluating the Visual Complexity of Chinese Ink Paintings
}

\author{
Zhen-BaO FAn ${ }^{1}$, Yi-Na Li ${ }^{2}$, Kang Zhang ${ }^{3}$, Jinhui Yu ${ }^{4}$ And MaO \\ LiN HUANG ${ }^{5}$
}

${ }^{1}$ Department of Computer Science, The University of Texas at Dallas, Richardson, TX 75082-3021 USA

${ }^{2}$ School of Computer Science, University of Technology Sydney, Ultimo NSW 2007. Australia

${ }^{3}$ Department of Computer Science, The University of Texas at Dallas, Richardson, TX 75082-3021 USA

${ }^{4}$ Department of Computer Science and Technology, Zhejiang University, Hangzhou, 310027 China

${ }^{5}$ School of Computer Science, University of Technology Sydney, Ultimo NSW 2007 Australia

Email:YINA.LI@student.uts.edu.au

\begin{abstract}
Painters arrange white space in contrast with chromatic space composed of strokes. This research measures white space, color complexity, and stroke density in Chinese ink paintings and examines how these attributes influence the paintings' perceived complexity. Empirical evidence from twenty-one well-known modern Chinese artists' ink paintings shows that white space decreases paintings' complexity, while chromatic space and stroke density increase complexity. We also reveal that a large rate of white space guides the viewers' attention on chromatic space and enhances the impacts of color complexity and stroke density on perceived complexity. An eye-tracker measures viewers' elaboration duration on each painting, which provides consistent evidence to validate our conclusion based on subjective reported visual complexity. Our research provides insights into the rhetorical role of white space in sensory information processing.
\end{abstract}

Keywords: Paintings, Complexity, Regression Model, Visual Rhetoric, Visual-Information Processing

\section{INTRODUCTION}

When a painting is being drawn, strokes are gradually added to a piece of paper, with part of the space filled by colors and the other part intentionally left blank. The chromatic space and its surrounding white space are the fundamental compositions of oriental paintings. The brushstrokes divide the dichotomic types of space on the paintings. As initially noted by Tao Te Ching (道 德经, in Chapter 11), the classical Taoist literature 1] regarding the utility of the emptiness and hollowness, an exploration of the dichotomic elements paves the way to an in-depth understanding of visual-information processing. Notably, the Taoist argument assigns equal weight to white space as to chromatic space, which conflicts with our cognitive intuition of the precedency of chromatic space, also called figure priority against the ground 22. As early as in Xi Zhou dynasty, recorded by Zhou Li . Kao Gong Ji (周礼 . 考工记), ancient Chinese painters indicated that highlighting the white space after chromatic coloring (凡画缋之事后素功) can improve the visual appeal of paintings. The basic logic of white and chromatic spatial categories ferments the subsequent visual convention of white space in Chinese paintings.

White space is widely used as a well-defined visual sign and as a stylistic element lacking clear meaning. For example, in Travelers Among Mountains and Streams (溪山行旅图) (see Fig. 1(a)d, the masterpiece of landscape by the well-known artist Fan Kuan (Song Dynasty, c.960 - c.1030), the white space on the right side of the painting (as highlighted by the zoom-in shot at top-left) is used to indicate a cascade as slender as silk falls from the heights above. Similarly, in the painting Wind Passing Pines in a Myriad of Valleys (万壑松 风图) (Fig. 1(b) by artist Li Tang (Song Dynasty, c.1050 - c.1130), the white space in the middle refers to the puffs of white clouds and concurrently serves as the background of rocks and trees. Li's exemplar shows the dual role of white space as a sign referring to a specific semantic meaning (i.e., the clouds) and as a stylistic element to accentuate other signs (i.e., the rocks and trees in this painting).

In line with the ancient philosophy of painting 


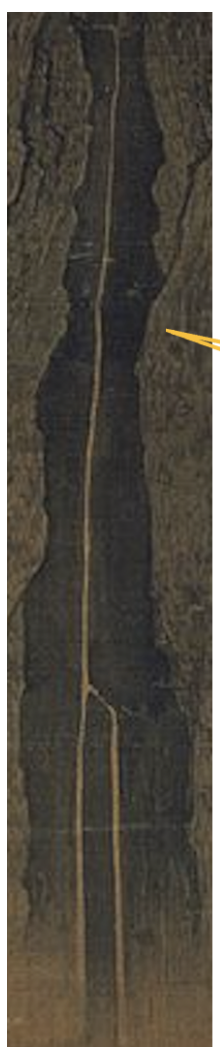

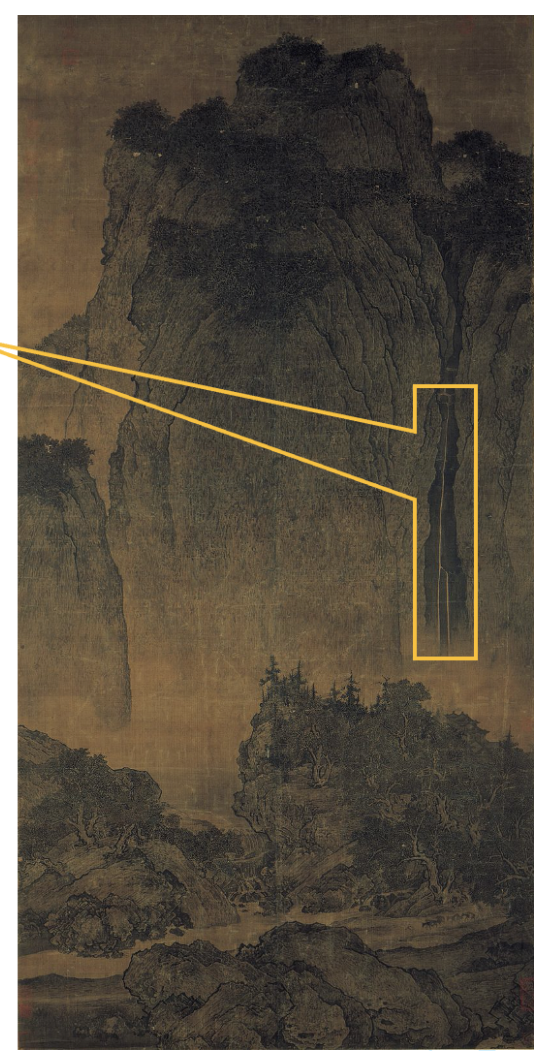

(a)

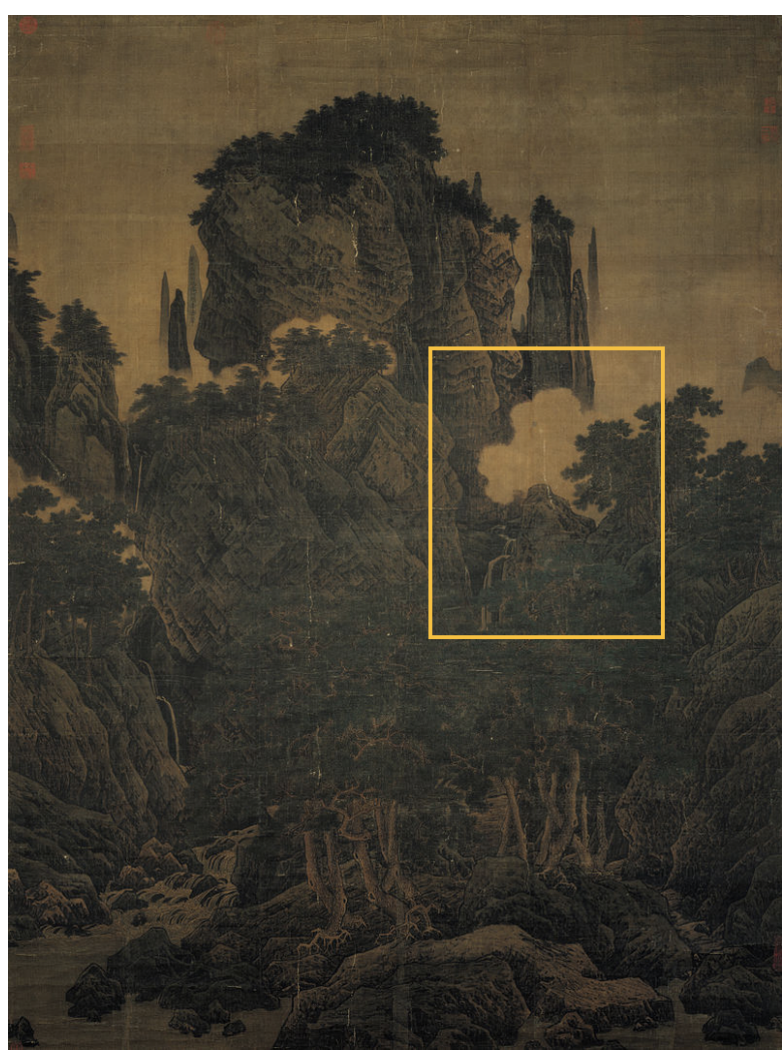

(b)

FIGURE 1. (a) Travelers Among Mountains and Streams. (b) Wind in Pines Among a Myriad Valley. Note: In the two paintings, the white spaces - originally left blank - has become brown on the oxidized paper.

composition based on the contrast of white and chromatic spaces, digital technology decomposes a painting into pixels which are either white or chromatic. Color science verifies that a color can be sufficiently and necessarily specified by a tri-component vector and vector space called "color space" 3]. The color of chromatic pixels can be defined by color spaces, which enables further applications of image-processing techniques to the measurements of color features, and in-depth quantitative research on the visual processing of paintings.

Viewers' perceived complexity is influenced by conceptual and perceptual complexity. Our research focuses on the perceptual complexity caused by sensory elements in painting images. The visual complexity of an image refers to the level of detail and intricacy contained in the image [4]. It is perceived by subjectively processing the objective attributes of the image. Perceived visual complexity influences the aesthetic appeal of an artwork and it is one of the most fundamental determinants of beauty [5] 6]. Our study explores how three visual attributes shape the perceived complexity of Chinese ink paintings. We select $221 \mathrm{ink}$ paintings from twenty-one well-known contemporary Chinese artists as samples. The samples are diversified in styles and include the most classical themes. We identify the impacts of white space, color complexity, and stroke density, on the perceived complexity by controlling the impacts of other attributes. An eyetracking experiment is further performed to validate our findings on how humans visually process paintings and other perceptual consequences (i.e., elaboration duration). White space is considered a conventionally defined rhetorical figure, and its interaction with chromatic space is discussed. Overall, our research contributes to the existing literature by

- Proposing quantified methods to gauge visual attributes that influence the complexity of paintings, including white space, color complexity, and stroke density;

- Providing empirical evidence on the impacts of white space, color complexity, and stroke density and their interactions on the perceived complexity of Chinese ink paintings;

- Shedding light on the role of white space as an exemplar of visual rhetoric in concentrating viewers' attention on chromatic space.

Our research consists of two studies. In Study 1, we collect multiple artists' ink paintings as samples. We measure the white space, color complexity, and 
stroke density of the paintings as independent variables and establish regression models to predict the perceived complexity of paintings. We also conduct a robustness check of our results by including additional control variables. In Study 2, we perform an eye-tracking experiment to collect empirical evidence on how people process white space and chromatic space, and perceive the visual complexity of the paintings.

In the remainder of this paper, Section 2 reviews related work on the role of complexity in aesthetic judgment. Section 3 states our hypotheses. Sections 4 identifies potential visual attributes that might influence perceived complexity and quantifies each attribute within a meaningful range. Sections 5 and 6 provide details of our two studies. Section 7 is conclusion and discussion, followed by limitation and future research in Section 8.

\section{RELATED WORK}

\subsection{Complexity in Aesthetic Judgment}

Visual complexity relates to the number and quality of basic visual elements, namely their dissimilarity and organization. The existing literature reviews the development of the idea of visual complexity and history of the measurement of complexity, from structuralism and Gestalt psychology to the visual complexity theory and perceptual learning theory $[7]$. Complexity is one of the most critical contributors in the measurement of beauty [8] 10. For example, Birkhoff's model of aesthetic measure includes complexity and order as two factors to determine beauty [11].

The existing literature addresses quantitative measurements of complexity on various objects, such as $2 \mathrm{D}$ and $3 \mathrm{D}$ shapes, web pages and photographs 12 13] 14. In artwork, aesthetic preference increases linearly with visual complexity until an optimum level is reached, and most of the studies agree that a moderate degree of complexity is preferred [6] 15. Particularly, existing research attempts to identify the influential factors of visual complexity and establish an assessment model on oil paintings [16], which are the most relevant work to our study.

\subsection{White space as visual rhetoric}

The human visual system processes figures with priority over backgrounds. By default, chromatic space is the figure and the unoccupied white space is the background. Chromatic shapes could attract viewers' attention to a greater extent earlier white space. White space, as a figure of visual rhetoric [17 18 [19], however, deviates from the most widely accepted convention. It can be more than space of unfilled-in 20 but supposed to indicate the semantic meaning of a vast, formless and ever-changing natural phenomenon, such as a sky, clouds, water, etc. [21]. As a distinguishing attribute defined by visual convention, white space is widely and deliberately adopted in paintings to evoke viewers' anticipation, activate their imagination, and invite their elaboration. The adoption of white space in Chinese ink paintings is highly appreciated after the literati painting blossoms in China's Ming dynasty (1364-1683). White space conveys high status through the linkage of the artists' high social status and illustrates the taste of the privilege.

Echoing the Chinese preliminary philosophy in art, modern minimalism introduces white space in visual design as a rhetorical figure 22] 23. Minimalists emphasize the "extreme economy of means" and the "essentials of geometric abstraction" in art and design, and encourage the belief "less is more" to be increasingly valued [22]. Such a belief is applied in architectural design and forms a clean look of upscale living 22 . It is also applied in graphic design to signal the diseconomy of information delivery and convey the "elegance, power and leadership" of high-end products 22 .

As a figure of visual rhetoric, white space, although developed in different cultural backgrounds and adopted in different genres of visuals, nonetheless conveys the meaning about high status consistently. Apart from our recent investigation [21], white space in paintings - as the precursor to modern visual rhetorical figures - is far from being well explored. The documented history of Chinese ink paintings rarely adopts empirical methods to examine how viewers perceive and interpret white space.

\subsection{Color complexity}

The human retina receives color stimuli in the preattentive stage, and the cerebral cortex combines the physical chromatic attributes and generates chromatic perception [24], including a large variety of hues. Color schemes are perceived at different levels of sensitivity and accuracy. Existing research explores the psychophysical relationship of chromatic attributes and their perception by measuring perceived color varieties, color variation, and color distribution in different color systems, and it examines their impacts on perceived color complexity.

Prior research assumes that perceived color varieties roughly reflect color complexity [25] and measures the colorfulness of a real-scene image by extracting a limited number of theme colors that represent the chromatic keynotes of the image [26]. Apart from the number of theme colors, the distributional characteristics of the color stimuli are addressed as the factors of color complexity. The spatial composition of colors (i.e., flat and polychrome compositions) is distinguished, and the color variations are measured by the distance of two pixels in the CIELab color space 27] 28]. 


\subsection{Stroke density}

Stroke is one of important components in painting. The size, shape, and color variation of brushstrokes and their compositions differ by painting styles, and humans can make aesthetic judgments by recognizing the way of using brushstrokes. Computer analysis of art explores marking and drawing tools (i.e., brushstrokes). Researchers use statistical patternrecognition techniques to learn the visual properties of brushstrokes that correspond to a particular painter or marking tool [29]. They apply the technique to analyze brushworks and identify artists, such as Vincent Van Gogh [30]. Also, they develop a stroke-based (SBR) rendering method that uses discrete strokes or stipples to represent visual objects and automatically creates non-photorealistic imagery 31], such as impressionistic paintings [32] and abstract images.

\section{HYPOTHESES}

A chromatically complex image provides more sensory input to generate percepts than a chromatically simple one. The coded chromatic pixels of a bitmap are the resources of information. As a lossy compression technique of still digital images, JPEG provides a method to store images with adjustable compression rates allowing the tradeoff of file size and image quality 33. In the existing literature, the JPEG file size of an image measures its total sensory input 34. Furthermore, a digital image of a real-world scene with a greater variety of colors is perceived as more complex than one with fewer colors 35], which we believe to be applicable to ink paintings. Therefore, we hypothesize that

H1: Color complexity increases perceived complexity.

White space can influence perceived complexity in two ways. On the one hand, as a stylistic element, white space provides limited sensory input, which requires little visual attention. Meanwhile, a large proportion of white space in an image excludes the potential accommodation of other complex visual contents. On the other hand, the visual convention of Chinese ink paintings assigns white space to objects [36]. The conventional use of white space provides a clear visual cue for organizing the sensory input, informing the visual objects; as such, it can greatly reduce the visual processing of semantic meanings. Therefore, we hypothesize that

H2: White space decreases perceived complexity.

Complexity refers to the level of details and intricacy of an object. As a basic element of painting, diversified strokes compose a painting. One stroke can be a thin and tiny point or a thick brush work covering large space. A high ratio of thin strokes produces perceived intricacy, while a few thick brushstrokes create a sense of simplicity. A complicated arrangement of strokes creates a complex layout and further increases visual complexity. Therefore, we assume that
H3: Stroke density increases perceived complexity.

As the simplest form of sensory input, white space provides a background in contrast to chromatic space. A large proportion of white space highlights the visual focus by enhancing the contrast of figure-ground discrimination. Therefore, the visual objects in the chromatic space would be more likely to attract viewers' attention. Consequently, their impacts on the perceived complexity of paintings would be strengthened. We hypothesize that

H4: White space moderates the impact of color complexity on perceived complexity. In other words, the impact of color complexity on perceived complexity is enhanced by a high proportion of white space.

Complexity attracts attention. A complex image provides more information for visual processing than a simple one. Perceived complexity activates the perceptual system to find regularities and seek for "simplicity in complexity" or "uniformity in variety" 37 [38. Existing literature in advertising images indicates that the complexity would increase viewers' elaboration duration 38]. Therefore, we propose that

H5: Perceived complexity elongates elaboration.

\section{COMPUTING COMPLEXITY}

This section describes our methods that quantify three independent variables (i.e., white space, color complexity, and stroke density) and control variables (i.e., hue count, aspect ratio, average saturation and lightness, balance score, mass center, and color contrast). The models in Section 5 would reveal the impacts of the three main variables on the perceived complexity and interaction effects between them.

\subsection{INDEPENDENT VARIABLES}

White Space. As a typical feature in traditional Chinese paintings, white space can be a stylistic expression and conventionally defined denotation. It contains a semantic meaning and typically represents a sky, cloud, river, or lake in scenic views, as the background of highlighted figures. It can also have an exclusively functional role as a style but indicating nothing concrete. Generally, the unoccupied area simplifies the composition of paintings more than those fully covered 36 . There could be many scattered small white pieces that are too small to be seen. White regions are more likely to be processed as meaningful objects and stylistic expressions than scattered white pieces, which are shown as trivial details, hardly influencing viewers' holistic impression of the paintings. Therefore, only sizable white regions on a painting are considered in our calculation of white space. We use a quadtree decomposition method 39 to capture white space in the paintings, which is proven effective in our previous study [36. As an example, Fig. 2 shows a painting, Boat, on which the calculated white space is outlined in red. 


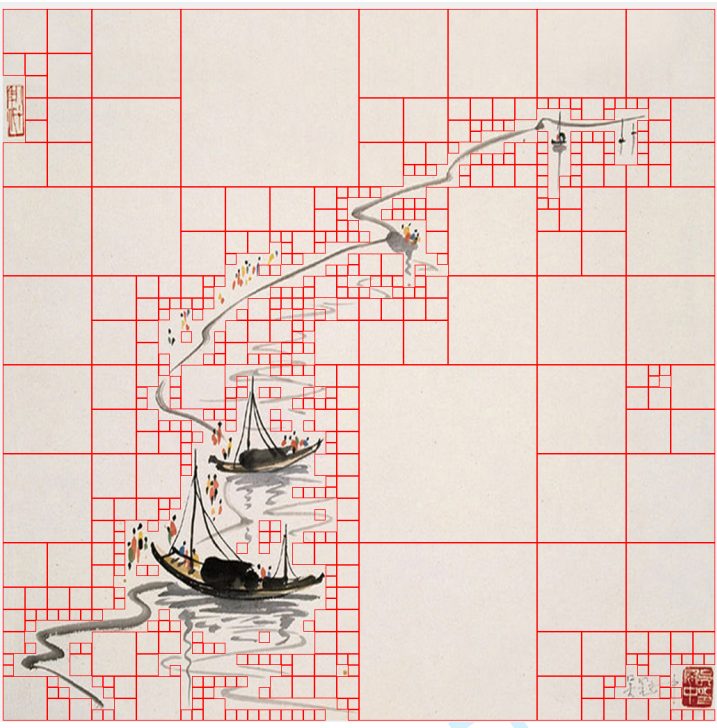

FIGURE 2. The calculation of white space in Boat. (Images processed by authors as fair use from wikiart.org)

Stroke Density. Stroke density represents the intricacy of paintings. One can visually distinguish thin strokes and tiny points from thick brush strokes in paintings. Generally, many thin strokes can increase perceived complexity, while a few thick strokes can decrease perceived complexity. Intuitively, a great number of color changes suggest many thin lines and small areas. We use a fast approach by simply calculating color changes among adjacent pixels vertically and horizontally and then computing the ratio of the number of changes over the total number of pixels in a painting image to measure its stroke density [36. Fig. 3 shows the calculated results using this method on Pine Tree, in which we mark a pixel in red if it has a different color from its top or left pixel.

Color Complexity. Apart from hue count, we also measure color complexity related to color variation and color distribution. Color Complexity Measurement (CCM) computes color intricacy of the chromatic space around each pixel 27]. It calculates the color pattern variation of each chromatic pixel by creating a local mask around each pixel and calculates the absolute deviation with Gaussian weighting within each mask. It defines the CCM value of a pixel at $(i, j)$ as 27 :

$$
\varphi(i, j)=\iint_{x, y \in \Omega_{(x, y)}} G_{\alpha}(\|c(x, y)-\bar{c}(i, j)\|) d x d y
$$

where $G_{\alpha}$ denotes the Gaussian weighting function and $\bar{c}(i, j)$ is the average color value within a local mask $\Omega(i, j)$ centered at $(i, j)$. \|\| denotes the color difference. CCM represents the color pattern variation around a pixel. A high CCM value means a high pattern variation among the neighboring pixels, while a small CCM value means that the region around this pixel is homogenous.

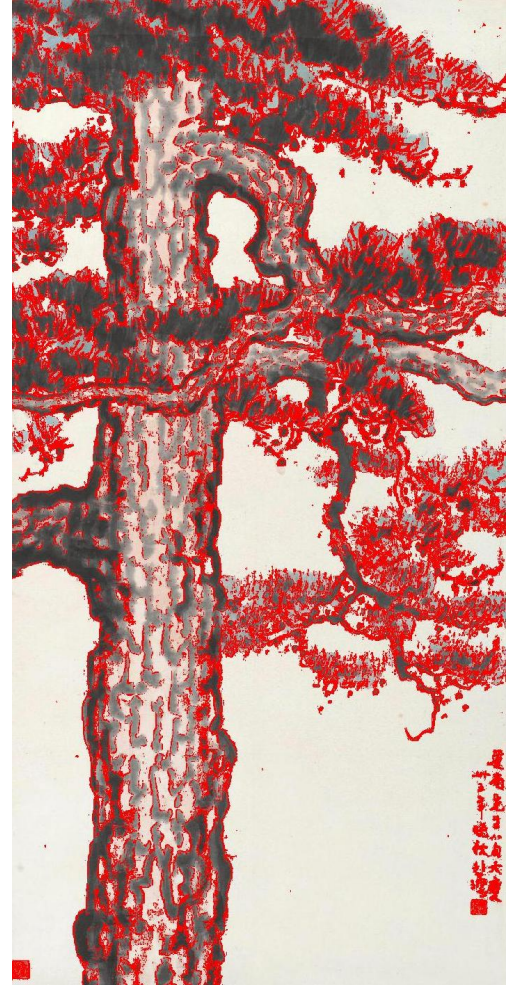

FIGURE 3. The calculation of stroke density on Pine Tree.

In the Gaussian mask of each pixel, the color difference between any two pixels is calculated by the Euclidean distance between the two points in the CIELab color space, which is proportional to the difference perceived by the human visual system. The RGB color components are first converted into CIEXYZ and then further converted to CIELab color components. The color difference in the CIELab color space is defined as 27]:

$$
D(c(i, j), c(x, y))=1-\exp \left(-\frac{E(c(i, j), c(x, y))}{\gamma}\right)
$$

where $\gamma$ is the normalized factor and $E(c(i, j), c(x, y))$ is the Euclidean distance in the CIELab color space.

Because the CCM value of each pixel implies its local complexity, the average of CCM values can be used to represent the color complexity of an entire painting, and it is obtained in the following steps: (1) Calculate the average color $\bar{c}(i, j)$ within the local mask of each pixel $c(i, j)$ in the given painting. (2) Calculate the color difference between the average $\bar{c}(i, j)$ and each pixel $c(x, y)$ within the local mask of pixel $c(i, j)$. (3) Calculate the CCM value of each pixel $c(i, j)$ using the absolute deviation with Gaussian weighting. (4) Calculate the average CCM value of all pixels to estimate the color complexity of the entire painting. Note that the white pixels are excluded by definition as the counterpart of the chromatic space. 


\subsection{CONTROL VARIABLES}

Hue Count. Color is one of the most intuitive features in human's visual processing. The number of colors in a painting is related to the perceived complexity of the painting. A painting using a few colors could look simpler than a polychrome one with many different colors. We calculate the number of different hues in each painting as follows. A color image is first converted into its HSV representation. Normally, only pixels with saturation $S>0.2$ and with brightness $0.15<V<0.95$ are considered effective pixels because pixels outside these ranges are possibly white, gray, or black 25] [40]. As described above in the calculation of white space, pixels with a saturation of below 20 and a brightness of over 80 tend to be white pixels. We also change 0.95 for $V$ to 0.8 here (i.e., we only consider pixels with a saturation of $S>0.2$ and a brightness of $0.15<V<0.8)$. A 20 -bin histogram $H$ is computed on the hue values of effective pixels and the hue count is defined as:

$$
N=\{i \mid H(i)>c m\}
$$

where $m$ is the maximum value of the histogram and $c$ is set at 0.35 because it produces a good result on our painting set. $N$ is the set of bins whose hue values are larger than $\mathrm{cm}$. The number of bins in $N$ represents the hue count.

Aspect Ratio. Chinese ink paintings have different types, such as hanging scrolls, screen paintings or normal types, and vary in aspect ratios. The aspect ratios of our selected paintings range from 0.296 to 4.119. Aspect ratio would potentially influence the viewer's assessment of the paintings.

Average Saturation and Lightness. Average saturation and lightness can more or less represent the color characteristics of a painting. Saturation represents the intensity, while lightness reflects the tone of a painting.

Balance score. Mass center is a position representing the balance point of the distributed mass in space. Paintings can be "well-balanced" or "poorlybalanced." In each painting, we calculate the "balance score" 41], as in the value $2 d / w$, in which $d$ is the distance of the mass center to the middle of the painting, and $w$ is the width of the picture. Balance score therefore has a range between -1 and +1 , where 0 indicates the position at the exact horizontal middle of the image, and -1 and +1 at the extreme left and right.

Distance of mass center to center. We calculate the Manhattan distance from the mass center to the image center in each painting. Because of the various sizes of the selected painting images, we consider the distance as $d 1 / w+d 2 / h$. Here, $d 1$ and $d 2$ are the distances to the image center of the painting on $\mathrm{x}$-axis and $\mathrm{y}$-axis, respectively, $w$ is the width, and $h$ is the height of the image.

Color Contrast. Each image is converted into its
HSL color representation. As with the calculation of white space, we only consider pixels with a saturation of $S>0.2$ and a lightness of $0.15<L<0.8$. The pixels outside these ranges are considered white, gray or black. A 20-bin histogram is computed on the hue values of effective pixels. In each bin, we obtain the center hue and calculate the maximum arc length on the hue wheel among 20 bins.

\section{STUDY 1: VISUAL COMPLEXITY OF PAINTINGS}

In this study, we empirically examine the impacts of the computed features on perceived complexity.

Procedure. We collect 221 contemporary Chinese ink paintings of twenty-one well-known artists, including Wu Guanzhong (1919-2010), Qi Baishi (1864-1957), Xu Beihong (1895-1953), Wu Hufan (1894-1968), Huang Binhong (1865-1955), Gao Jianfu (1879-1951), Liu Haisu (1896-1994), Dong Shouping (1904-1997), Li Keran (1907-1989), Guan Shanyue (1912-2000), Wu Zuoren (1908-1997), Zhang Daqian (1899-1983), Li Kuchan (1899-1983), Lin Fengmian (1900-1991), Pan Tianshou (1897-1971), Hu Peiheng (1892-1965), Zhao Shao'ang (1905-1998), Zhao Wangyun (1906-1977), Chen Shaomei (1909-1954), Huang Qiuyuan (1914-1979), and Li Xiongcai (1910-2001). Their paintings inherit traditional techniques in strokes and composition, and they also create modern Eastern styles. We recruit fifty participants from a Chinese online panel. Each participant is assigned twenty paintings for at least 5 seconds on a computer monitor and asked to rate his/her perceived complexity of the paintings $(1=$ extremely simple; $7=$ extremely complex). To guarantee consistent and clear understanding of complexity evaluation, we explain the definition of visual complexity to the participants as in Section 2.1. We also show the participants several sample paintings diversified in visual complexity, to illustrate how the number of visual elements, the similarity of visual objects, and organizations of the elements lead to the difference in visual complexity. None of the participants is color blind or an art professional.

Results. Table 1 presents the descriptive statistics and correlations between computed variables. We establish linear regression models using the perceived complexity of each painting as the dependent variable and the computed features as independent variables. The results in Table 2 show that high color complexity and stroke density increase the perceived complexity (supporting H1 and H3), while a large proportion of white space decreases the perceived complexity (supporting H2). We use hue count, aspect ratio, average saturation and lightness, balance score, distance of mass center to center, and color contrast as control variables. Model 1 contains only the control variables. Model 2 includes the main effect of white 


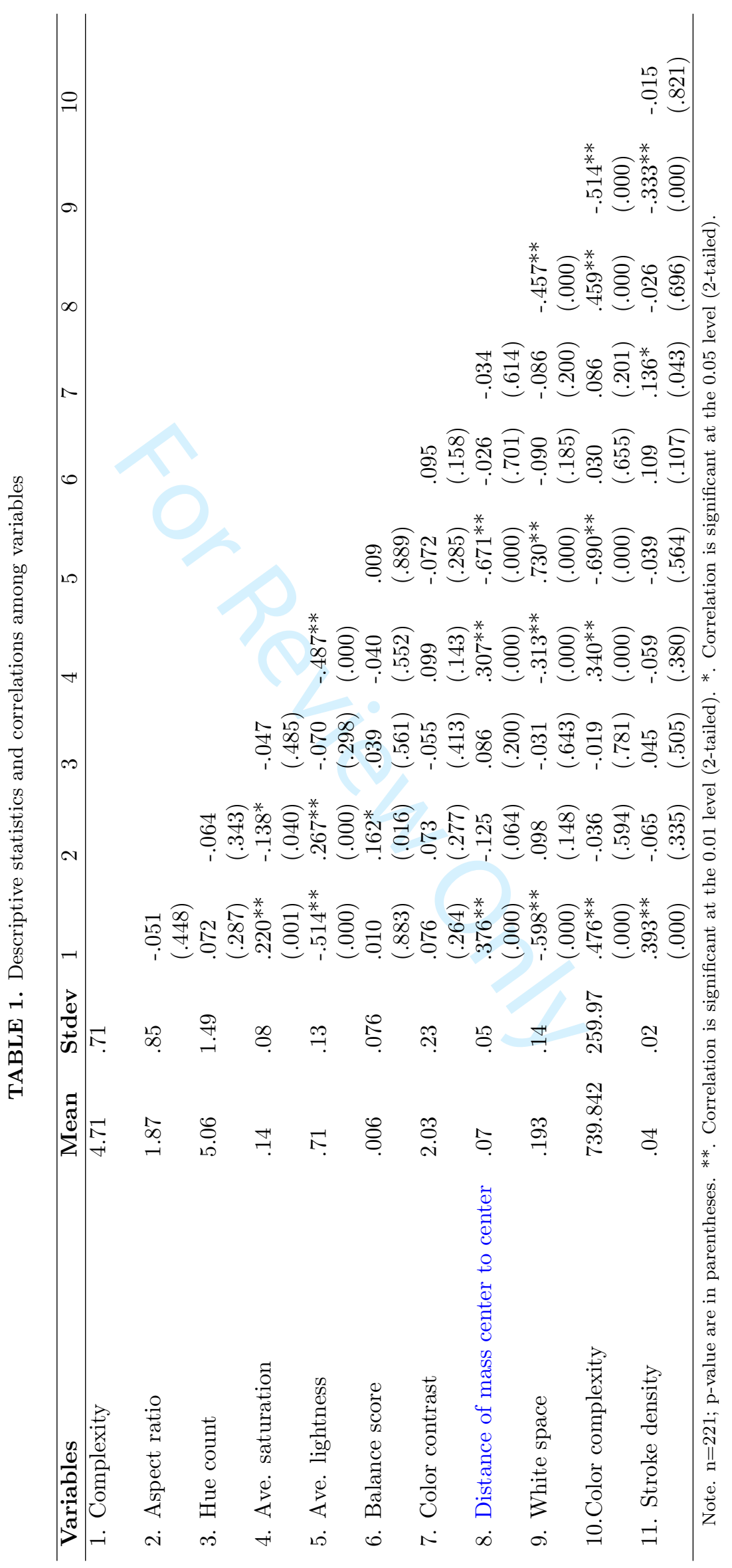


space, color complexity, and stroke density. Models 3 and 4 include the two interaction effects, respectively. Model 5 is the full model that includes all of the main and interaction effects.

Model 2 tests the effects of three main variables and increases $R^{2}$ from 0.289 in Model 1 to 0.501 . White space is significantly and negatively associated with perceived complexity $(p=0.000)$, supporting Hypothesis 2, which holds that white space decreases complexity. Color complexity and stroke density have a positive and significant effect $(p=0.002$ and $p=0.000$, respectively), supporting Hypotheses 1 and 3, which maintain that high color complexity and high stroke density increase the perceived complexity.

Model 3 tests the interaction between color complexity and white space. The positive interactive effect of white space and color complexity on perceived complexity $(p=0.027)$ confirms that a large proportion of white space enhances the impact of color complexity on perceived complexity (supporting Hypothesis 4). Similarly, Model 4 reveals the positive interactive effect of white space and stroke density $(p=0.029)$ on perceived complexity, confirming that the contrast of chromatic space to a large white space enhances the impact of stroke density on perceived complexity. Model 5 is the full model.

Discussion. The linear regression models provide evidence on the positive effect of color complexity and stroke density, and the negative effect of white space on the perceived complexity of Chinese ink paintings. The moderation effect of white space and color complexity on perceived complexity reveals that white space can be used as an intentionally compositional arrangement to guide the viewers' attention such that they focuses on chromatic space. We also find consistent evidence from the moderation effect of white space and stroke density on perceived complexity, which suggests a similar role of white space. Both of the interactive effects support the categorization of white and chromatic spaces.

\section{STUDY 2: IMPACT OF COLOR COM- PLEXITY ON ELABORATION USING EYE-TRACKING DATA}

We use the eye-tracking technique to observe how people view paintings. People would pay more attention to images of high complexity. We measure their total fixation duration in processing of each painting as the evidence to show the validity of the self-reported perceived complexity. We assume that high complexity requires viewers to spend a longer duration to elaborate a large number of visual objects and details [42. Also, we analyze the durations of fixation on the paintings and provide evidence to support our argument in respect of the role of white space in manipulating the viewers' attention.

Procedure. A total of 99 participants at a major university were recruited to randomly view 10 of 30
Chinese ink paintings and to rate the complexity of the paintings using the same measurement of perceived complexity as in Study 1. We collect eye-tracking data to explore the impact of color complexity on viewers' elaboration. A high frequency $(60 \mathrm{~Hz})$ eye tracker (Tobii T60) with two infrared image sensors can catch and record eye movements when viewers observe the paintings. All the participants were required to complete a calibration process to ensure that the device records their eye movements accurately.

The behavior of eye movements can be categorized as rapid saccades (scan) and fixations (gaze) 43. When a viewer gazes at a certain area that is relatively stable, the fixation duration is usually used to measure the elaboration of the visual information in this area. We adopt the total fixation duration of the entire picture as the measurement of attention.

Results. The descriptive statistics reveal that the mean value of the ratio of white space is 0.122 , with $S D=0.176$; the average color complexity is 1190.761 , with $S D=668.789$; the average complexity of the paintings is 3.684 , with $S D=1.199$; and the average total fixation duration on the paintings is 5.20 seconds, with $S D=0.475$. We adopt the $\log$ transferred value of the ratio of white space. Linear regression shows that stroke density increases perceived complexity $(B=2.088$, Std.Error $=1.171, p=0.086)$, color complexity increases perceived complexity $(B=$ 0.337, Std.Error $=0.148, p=0.031)$, and white space decreases perceived complexity $(B=-2.397$, Std.Error $=0.933, p=0.016)$, with $F=14.801$, $R^{2}=0.631$ and adjusted $R^{2}=0.588$. We also conduct a moderated mediation analysis using SPSS MACRO PROCESS Model 7 (bootstrapping samples = $5,000)$, controlling semantic clarity. As the result, color complexity increases complexity $(b=8-4 e, p=0.022)$, large white space decreases complexity $(b=-0.121$, $p=0.015)$, and the positive effect of color complexity on perceived complexity is enhanced by large white space $(b=1-4 e, p=.036)$. Perceived complexity marginally increases total fixation duration $(b=0.277, p=.091)$. The results confirm a moderated mediation effect of color complexity on total fixation duration through perceived complexity $(90 \% C I: 9-4 e, 452-4 e)$. Specifically, a high color complexity increases perceived complexity and consequently leads to a long elaboration (measured by the total fixation duration of the entire painting), which is in line with H5. The impact of color complexity is fully mediated by perceived complexity. White space moderates the impact of color complexity on perceived complexity (H4), meaning that the impact of color complexity on perceived complexity is enhanced when the area of white space is large. Fig. 5 further illustrates the interactive effect of color complexity and white space on perceived complexity. The moderated mediation effect on total fixation duration is significant when a painting contains a large amount of white space.

Additional evidence on visual processing. We 


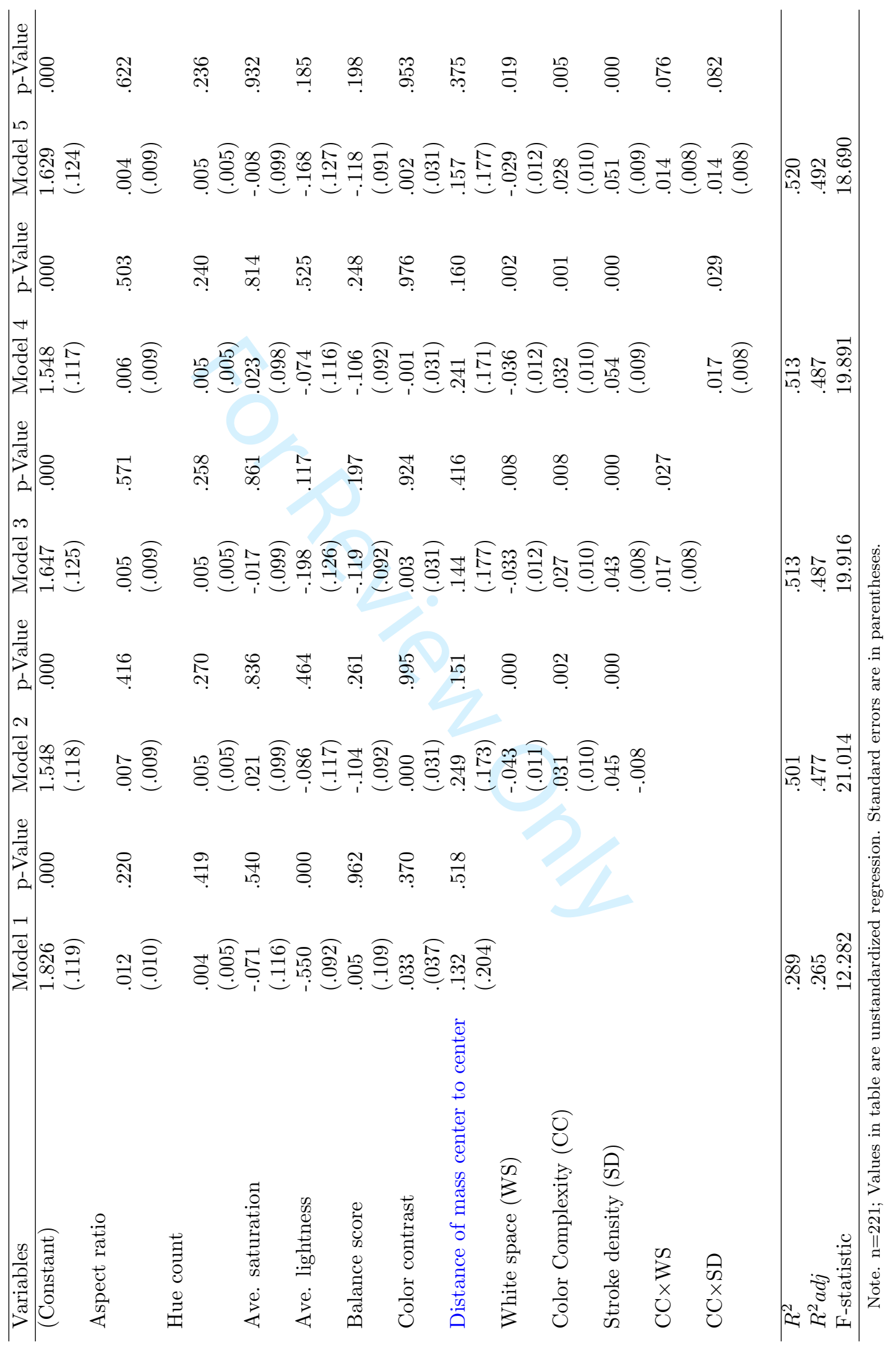




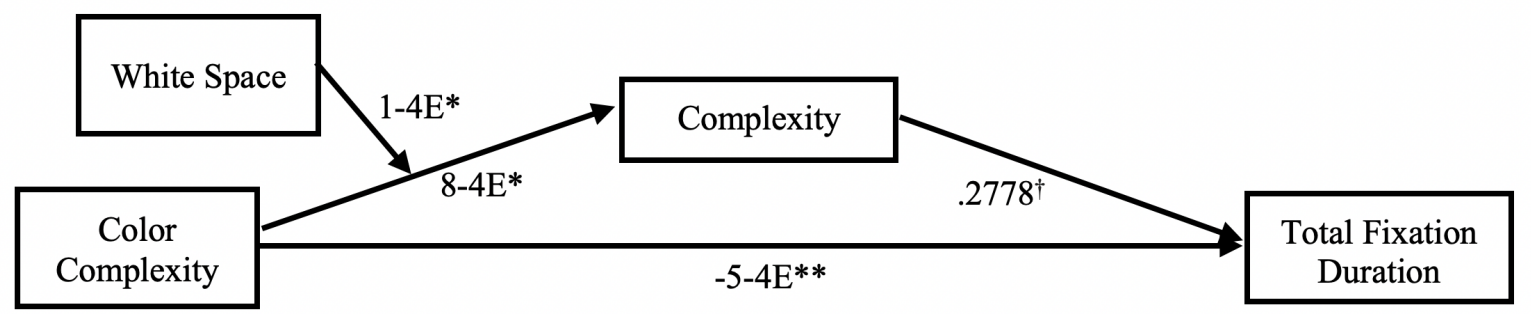

FIGURE 4. Mediation results of visual complexity.

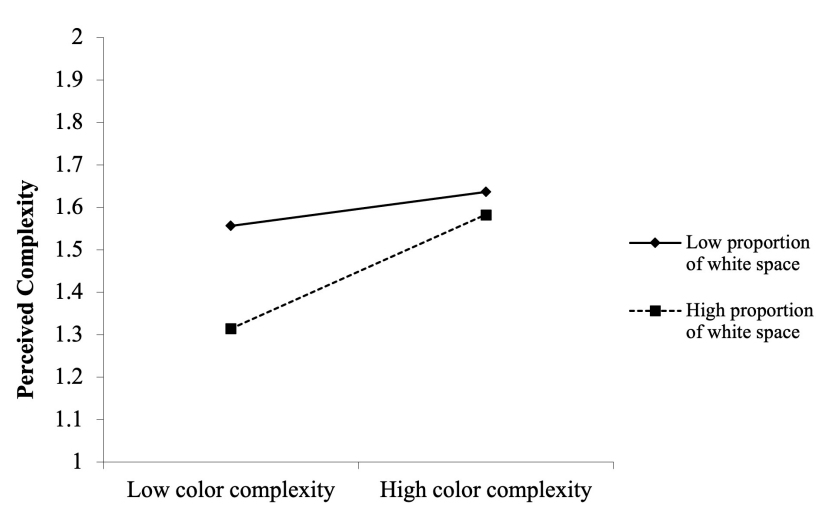

FIGURE 5. The interactive effect of color complexity and white space on perceived complexity.

Note: In this figure, the dashed line has a steeper slope than the solid line, suggesting that the effect of color complexity is stronger when a painting contains a high proportion of white space than a low proportion of white space.

analyze the eye behavior on each painting. The visualization intuitively reveals the attribution of visual attention on white space, chromatic space, and strokes, apart from the statistics-based evidence.

The heat maps in Fig. 6(a) $6(\mathrm{~b}) \mid[(\mathrm{c})$ and $6(\mathrm{~d})$ show that viewers pay attention to chromatic spaces and strokes, as the theory of priority of figure (vs. background) argues [44. For example, the boats in Boats and buildings in Riverside Town earn viewers' attention. The heat maps in Fig. 6(e) and 6(f) show that more attention is paid to the center of the painting than to other places. There is increased information processing on chromatic areas and strokes when the white space is large. Large white space can increase the impacts of color complexity and stroke density. This observation is in line with our statistics-based evidence above.

As white space is perceptually processed as irregular, it invites an explanation about the irregularity. White space in paintings is recognized as a stylistic element or meaningful visual object. As its semantic meaning is evoked, white space becomes the vehicle of conveying meaning. Theoretically, one might argue that when white space refers to clear semantic meanings, such as the lake in Fig 6(a), it is possibly processed equally as the chromatic objects. However, eye-tracking data refutes this argument. Viewers do not gaze at white space and interpret its denotation. Instead, they are more likely to infer the semantic meaning of visual objects in white space based on the chromatic visual cues. White space enhances the priority of chromatic attributes and strokes.

Discussion. The results provide evidence for the impact of color complexity on viewers' appreciation of perceptual complexity. The moderated mediation effect of color complexity and white space provides an insight into the understanding of the fundamental principle in visual art, namely the interactive influences of white and chromatic spaces. Considering two paintings with the same level of color complexity, the one with large white space would be perceived as having a higher level of complexity and, accordingly, would be processed with a longer duration. Thus, it is the absence of color that enhances the elaboration of the presence.

\section{CONCLUSION AND DISCUSSION}

Our study views white space and chromatic space that is composed of strokes as the main components in Chinese ink paintings and gauges visual attributes that are directly related to complexity in these three aspects. We provide empirical evidence including regression models and eye tracking experiment and indicate the positive effect of color complexity (H1) and stroke density (H3), as well as and the negative effect of white space (H2) on perceived complexity. Also, we reveal the interactive effects of white space and chromatic space on perceived complexity (H4) and validate the reported perceived complexity using the elaboration duration on each painting (H5). Our research offers the following contributions to the literature.

First, our research gauges visual attributes that influence the complexity of paintings and provides an objective view to understand viewers' visualinformation processing. Compared to the existing research on the objective measurement of paintings, which identifies color attributes as influential variables on the complexity of oil paintings [16], our research provides evidence from different genres of paintings and reveals the link between the sensory elements and 


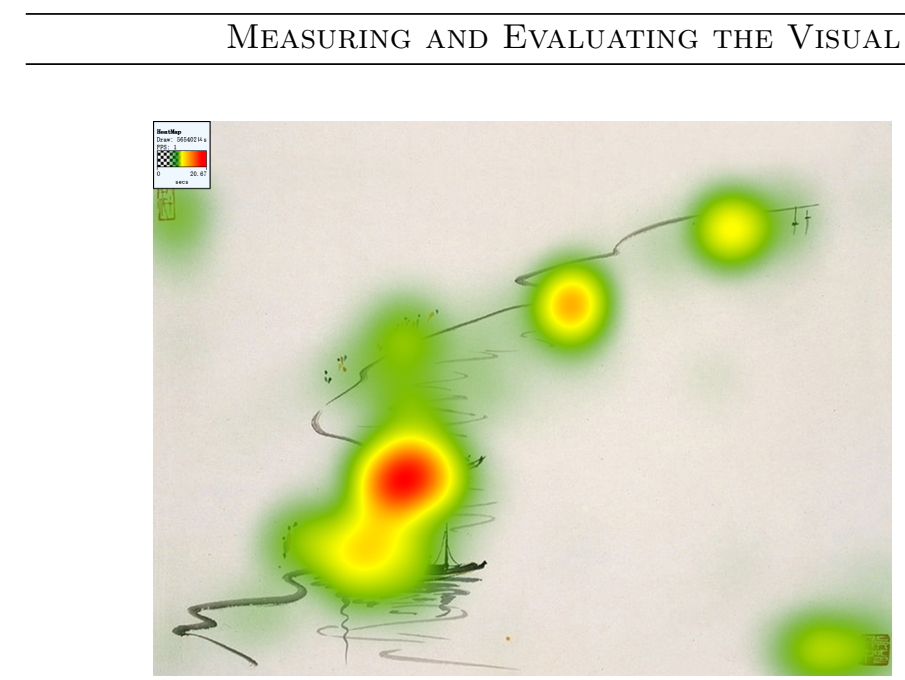

(a)

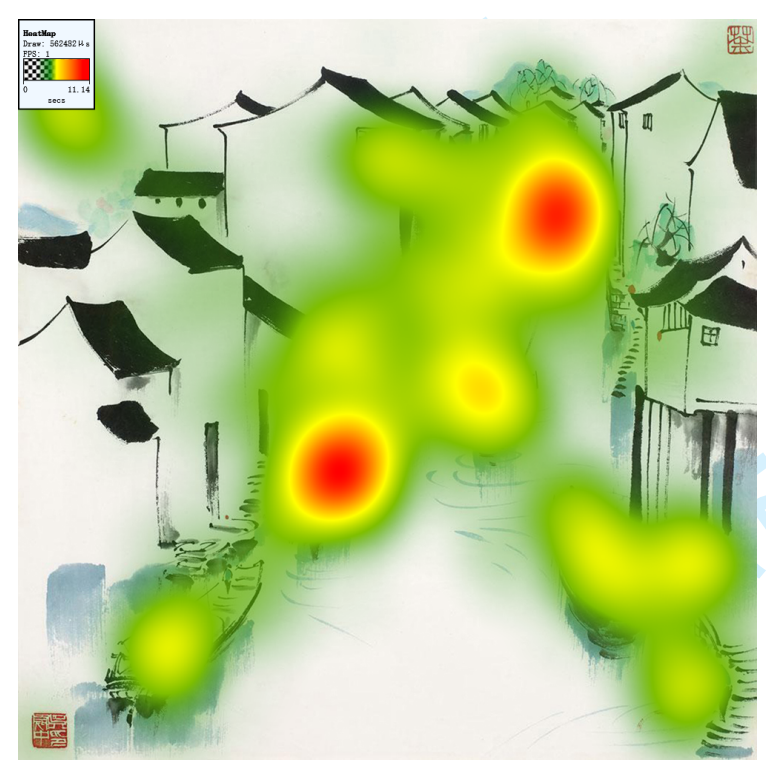

(c)

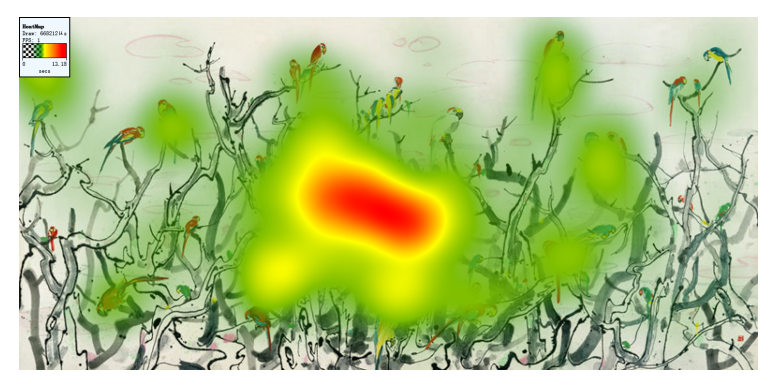

(e)

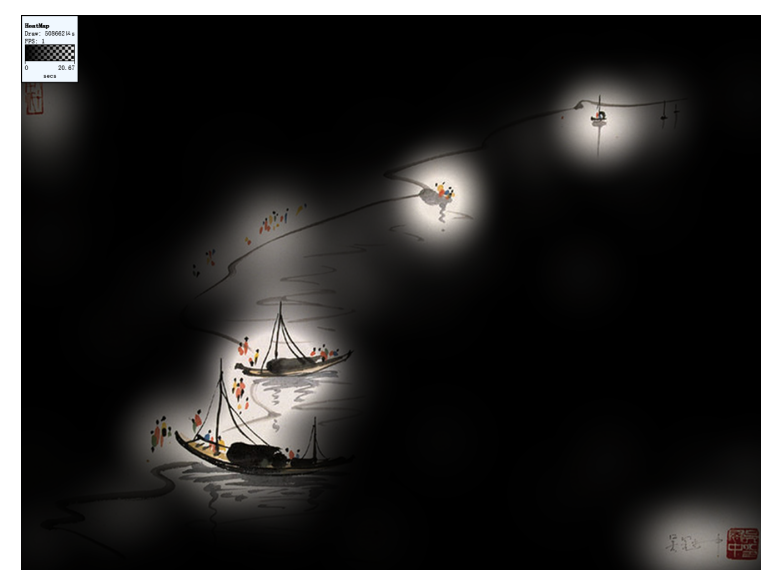

(b)

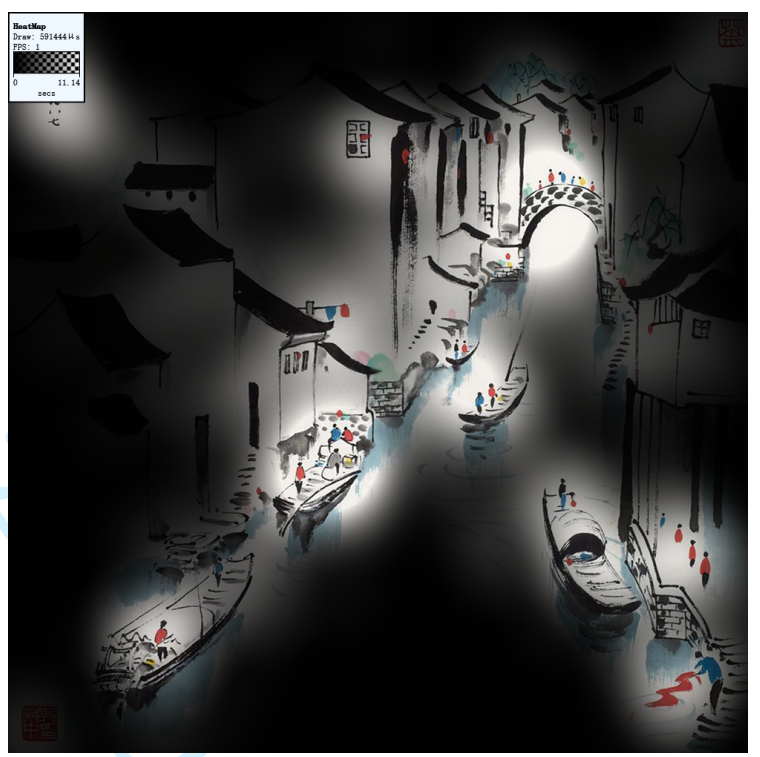

(d)

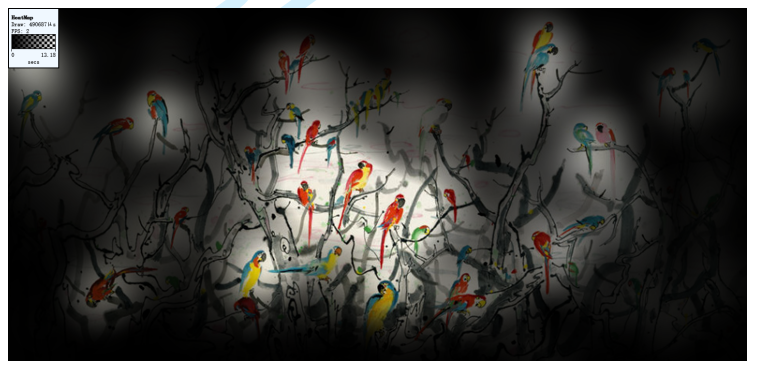

(f)

FIGURE 6. Heat maps of fixation duration on three Chinese ink paintings, (a)(b): Boats, (c)(d): Riverside Town, (e)(f): Heaven of Parrots.

perceived complexity.

Second, we attempt to explore the impacts of physical attributes on a type of aesthetic judgment (i.e., complexity). We provide empirical evidence on how sensory elements are perceived and organized to cause the difference in experienced complexity 7 . The categorization of white space and chromatic space provides an insight to explore the processing of two types of sensory information and their interaction. Apart from the main effects of white space, chromatic space, and stroke density, we reveal the interactive effects of white space and chromatic space on perceived complexity. The results supporting $\mathrm{H} 4$ reinforce that, as the sensory input emphasizing the visual priority 
of chromatic space, a large proportion of white space enhances the impact of color complexity on perceived complexity.

Third, our research sheds light on the understanding of white space as visual rhetoric in painting appreciation. Unlike the existing research that focuses on chromatic space, explores the measurement of random polygons [45], and investigates the computational aesthetic evaluation of logos [46], our research highlights white space as the counterpart of chromatic space. We indicate the dual role of white space as both a sensory input of stylistic elements and a symbol referring to real-world objects. By gauging the proportion of white space in well-known Chinese ink paintings using an algorithm (rather than manipulating a limited number of images, as in the existing literature), our research addresses blankness as a sign, and it extends the semiotic theories of white space to the context of Chinese aesthetics. The findings in this paper, which focus on a specific genre within a specific culture, namely Chinese ink paintings, provide a good starting point for examining "white space" in visuals in other genres (advertising, political cartoons, manuals, etc.) and in other cultures (British Pop Art, French Impressionism, Russian Constructivism, etc.).

\section{LIMITATION AND FUTURE RESEARCH}

Both conceptual and perceptual complexity increase viewers' perceived complexity. Our research focuses on the perceptual complexity caused by sensory elements including color complexity, stroke density and white space. We choose samples of Chinese ink paintings in which the landscape scenery, birds, flowers, and plants are similar in respect of in conceptual complexity, but diversified in perceptual complexity. Due to the limited themes of the selected samples, our conclusions are unable to gauge the conceptual complexity of paintings, such as that of narrative paintings. Future research could explore how the plot of a story at a particular moment is displayed and reveal the factors of conceptual complexity.

It should be prudent to balance the white and chromatic spaces of a particular painting based on our estimated effects. The sensory factors of visual complexity, as our research reveals, does not promise a golden standard that is universally applicable to each Chinese ink painting. An artist endeavors to find the best match between the form and content of a painting. To create an interpretable artwork, the adoption of stylistic elements depends on the theme and content, which limits the freedom with which an artist could adjust the ratio of white space to chromatic space. Future research could explore the favorable ratios for particular types of content and thereby propose a guideline for adopting of the stylistic elements.

\section{ACKNOWLEDGEMENTS}

This research is supported by National Natural Science Foundation of China (72072169, 71702080, 61772463), Humanities and Social Sciences Fund of Ministry of Education (17YJC630071), and an Australian Government Research Training Program.

\section{DATA AVAILABILITY STATEMENT}

The data that support the findings of this study are available from the author, Zhen-Bao Fan, upon reasonable request.

\section{REFERENCES}

[1] Tzu, L. (1992) Tao Te Ching translated by Stephen Mitchell. HarperCollins, New York.

[2] Poort, J., Raudies, F., Wannig, A., Lamme, V. A., Neumann, H., and Roelfsema, P. R. (2012) The role of attention in figure-ground segregation in areas v1 and v4 of the visual cortex. Neuron, 75, 143-156.

[3] Plataniotis, K. N. and Venetsanopoulos, A. N. (2000) Color image processing and applications. SpringerVerlag, Berlin.

[4] Forsythe, A. (2009) Visual complexity: is that all there is? International Conference on Engineering Psychology and Cognitive Ergonomics, San Diego, CA, 19-24 July, pp. 158-166. Springer, Berlin.

[5] Nadal, M., Munar, E., Marty, G., and Cela-Conde, C. J. (2010) Visual complexity and beauty appreciation: Explaining the divergence of results. Empirical Studies of the Arts, 28, 173-191.

[6] Forsythe, A., Nadal, M., Sheehy, N., Cela-Conde, C. J., and Sawey, M. (2011) Predicting beauty: fractal dimension and visual complexity in art. British journal of psychology, 102, 49-70.

[7] Donderi, D. C. (2006) Visual complexity: a review. Psychological bulletin, 132, 73-97.

[8] Frith, C. D. and Nias, D. K. (1974) What determines aesthetic preferences. Journal of General Psychology, 91, 163-173.

[9] Leder, H., Belke, B., Oeberst, A., and Augustin, D. (2004) A model of aesthetic appreciation and aesthetic judgments. British journal of psychology, 95, 489-508.

[10] Tatarkiewicz, W. (1999) History of Aesthetics. Bloomsbury Publishing PLC, London.

[11] Birkhoff, G. D. (1933) Aesthetic measure. Harvard University Press, Cambridge.

[12] Harper, S., Jay, C., Michailidou, E., and Quan, H. (2013) Analysing the visual complexity of web pages using document structure. Behaviour \& Information Technology, 32, 491-502.

[13] Perkiö, J. and Hyvärinen, A. (2009) Modelling image complexity by independent component analysis, with application to content-based image retrieval. International Conference on Artificial Neural Networks, Limassol, Cyprus, 14-17 September, pp. 704-714. Springer, Berlin.

[14] Psarra, S. and Grajewski, T. (2001) Describing shape and shape complexity using local properties. Proceedings 3rd International Space Syntax Symposium, 
Atlanta, 7-11 May, pp. 28:1-28:16. University of Michigan, Ann Arbor.

[15] Berlyne, D. E. (1973) Aesthetics and psychobiology. Appleton-Century-Crofts, Norwalk.

[16] Guo, X., Kurita, T., Asano, C. M., and Asano, A. (2013) Visual complexity assessment of painting images. 2013 IEEE International Conference on Image Processing (ICIP), Melbourne, 15-18 September, pp. 388-392. IEEE, New York.

[17] McQuarrie, E. F. and Mick, D. G. (1996) Figures of rhetoric in advertising language. Journal of consumer research, 22, 424-438.

[18] McQuarrie, E. F. and Mick, D. G. (1999) Visual rhetoric in advertising: Text-interpretive, experimental, and reader-response analyses. Journal of consumer research, 26, 37-54.

[19] McQuarrie, E. F. and Mick, D. G. (2003) Visual and verbal rhetorical figures under directed processing versus incidental exposure to advertising. Journal of consumer research, 29, 579-587.

[20] White, A. W. (2002) The elements of graphic design: space, unity, page architecture, and type. Allworth Press, New York.

[21] Fan, Z.-B., Zhang, K., and Zheng, X. S. (2019) Evaluation and analysis of white space in wu guanzhong's chinese paintings. Leonardo, 52, 111-116.

[22] Pracejus, J. W., Olsen, G. D., and O'Guinn, T. C. (2006) How nothing became something: white space, rhetoric, history, and meaning. Journal of Consumer Research, 33, 82-90.

[23] Pracejus, J. W., O'Guinn, T. C., and Olsen, G. D. (2013) When white space is more than "burning money": Economic signaling meets visual commercial rhetoric. International Journal of Research in Marketing, 30, 211-218.

[24] Gegenfurtner, K. R. and Kiper, D. C. (2003) Color vision. Annual review of neuroscience, 26, 181-206.

[25] Li, C. and Chen, T. (2009) Aesthetic visual quality assessment of paintings. IEEE Journal of selected topics in Signal Processing, 3, 236-252.

[26] Morse, B. S., Thornton, D., Xia, Q., and Uibel, J. (2007) Image-based color schemes. 2007 IEEE International Conference on Image Processing, San Antonio, TX, 16 Sept.-19 Oct, pp. III:497-III:500. IEEE, New York.

[27] Yoon, K.-J. and Kweon, I.-S. (2001) Color image segmentation considering human sensitivity for color pattern variations. Proceedings of SPIE, Boston, MA, 5 October, pp. 269-279. SPIE, Bellingham.

[28] Wan, X. and Kuo, C.-C. J. (1996) Color distribution analysis and quantization for image retrieval. Storage and Retrieval for Still Image and Video Databases IV, 13 March, pp. 8-16. SPIE, Bellingham.

[29] Stork, D. G. (2009) Computer vision and computer graphics analysis of paintings and drawings: An introduction to the literature. International Conference on Computer Analysis of Images and Patterns, Münster, Germany, 2-4 September, pp. 9-24. Springer, Berlin.

[30] Johnson, C. R., Hendriks, E., Berezhnoy, I. J., Brevdo, E., Hughes, S. M., Daubechies, I., Li, J., Postma, E., and Wang, J. Z. (2008) Image processing for artist identification. IEEE Signal Processing Magazine, 25, $37-48$.

[31] Hertzmann, A. (2003) A survey of stroke-based rendering. IEEE Computer Graphics and Applications, 4, 70-81.

[32] Haeberli, P. (1990) Paint by numbers: Abstract image representations. Computer Graphics (SIGGRAPH '90 Proceedings), 24, 207-214.

[33] Haines, R. F. and Chuang, S. L. (1992) The effects of video compression on acceptability of images for monitoring life sciences experiments. National Aeronautics and Space Administration, Moffett Field, CA.

[34] Pieters, R., Wedel, M., and Batra, R. (2010) The stopping power of advertising: Measures and effects of visual complexity. Journal of Marketing, 74, 48-60.

[35] Olivia, A., Mack, M. L., Shrestha, M., and Peeper, A. (2004) Identifying the perceptual dimensions of visual complexity of scenes. Proceedings of the 26th Annual Meeting of the Cognitive Science Society, Chicago, Illinois, 4-7 August, pp. 1041-1046. Cognitive Science Society, Seattle.

[36] Fan, Z.-B., Li, Y.-N., Yu, J., and Zhang, K. (2017) Visual complexity of chinese ink paintings. Proceedings of the ACM Symposium on Applied Perception, Cottbus, September, pp. 1-8. Association for Computing Machinery, New York.

[37] Dickie, G. (1997) Introduction to aesthetics: An analytic approach. Oxford University Press, Oxford.

[38] Reber, R., Schwarz, N., and Winkielman, P. (2004) Processing fluency and aesthetic pleasure: Is beauty in the perceiver's processing experience? Personality and social psychology review, 8, 364-382.

[39] Finkel, R. A. and Bentley, J. L. (1974) Quad trees a data structure for retrieval on composite keys. Acta informatica, 4, 1-9.

[40] Ke, Y., Tang, X., and Jing, F. (2006) The design of high-level features for photo quality assessment. 2006 IEEE Computer Society Conference on Computer Vision and Pattern Recognition (CVPR'06), New York, NY, 17-22 June, pp. 419-426. IEEE, New York.

[41] McManus, I., Edmondson, D., and Rodger, J. (1985) Balance in pictures. British Journal of Psychology, 76, 311-324.

[42] Just, M. A. and Carpenter, P. A. (1976) Eye fixations and cognitive processes. Cognitive psychology, 8, 441480.

[43] Holmqvist, K., Nyström, M., Andersson, R., Dewhurst, R., Jarodzka, H., and Van de Weijer, J. (2011) Eye tracking: A comprehensive guide to methods and measures. Oxford University Press, Oxford.

[44] Castro, L., Lazareva, O. F., Vecera, S. P., and Wasserman, E. A. (2010) Changes in area affect figureground assignment in pigeons. Vision research, 50, 497-508.

[45] Mavrides, C. M. and Brown, D. (1969) Discrimination and reproduction of patterns: Feature measures and constraint redundancy as predictors. Perception 83 Psychophysics, 6, 276-280.

[46] Zhang, J., Yu, J., Zhang, K., Zheng, X. S., and Zhang, J. (2017) Computational aesthetic evaluation of logos. ACM Transactions on Applied Perception (TAP), 14, $1-21$. 